\title{
The Spatio-Temporal Variation Characterization of Ecological Drought in the Yangtze River Source Region, China Using a Standardized Water Supply-Demand Index
}

\author{
Yuan Zhe ${ }^{1}$, Jijun $\mathrm{Xu}^{1}$, Zhiguang $\mathrm{Wu}^{1}$, Yongqiang Wang ${ }^{1}$, and Shu Chen ${ }^{1}$ \\ ${ }^{1}$ Changjiang River Scientific Research Institute
}

May 5, 2020

\begin{abstract}
It is important for drought risk assessment and sustainable development of water resources on the basis of understanding the spatio-temporal characteristics of drought and return period. This study introduced a new drought index, standardized supply-demand water index (SSDI), and a run theory which are applied to identify ecological drought events and parameters (e.g. duration, severity, peak and coverage area) in the Yangtze River Source Region (YRSR). And the bivariate probability and return period were calculated via 2-dimensional joint copula to investigate the drought-prone regions. The results indicate that: (1) Compared with traditional meteorological drought index, the SSDI is reliable and can reflect the comprehensive characteristics of the ecological drought information more easily and effectively; (2) The YRSR had witnessed the most severe drought episodes in the periods of late-1970s, mid-1980s and mid-1990s, but the SSDI showed a wetting trend since mid-2000s. And droughts in the Southern YRSR were relatively more severe with longer drought duration; (3) In most areas of Togton River Basin and Dam River Basin, the severe ecological drought events happened more frequently; (4) Drought duration and severity in the YRSR were more susceptible to temperature when the temperature rise were above $1.0^{\circ} \mathrm{C}$. The average drought duration and severity increased by $20.7 \%$ and $32.6 \%$ with a temperature rise of $1^{\circ} \mathrm{C}$.
\end{abstract}

\section{Hosted file}

00Manuscript.pdf available at https://authorea.com/users/302574/articles/432681-the-spatiotemporal-variation-characterization-of-ecological-drought-in-the-yangtze-river-sourceregion-china-using-a-standardized-water-supply-demand-index 


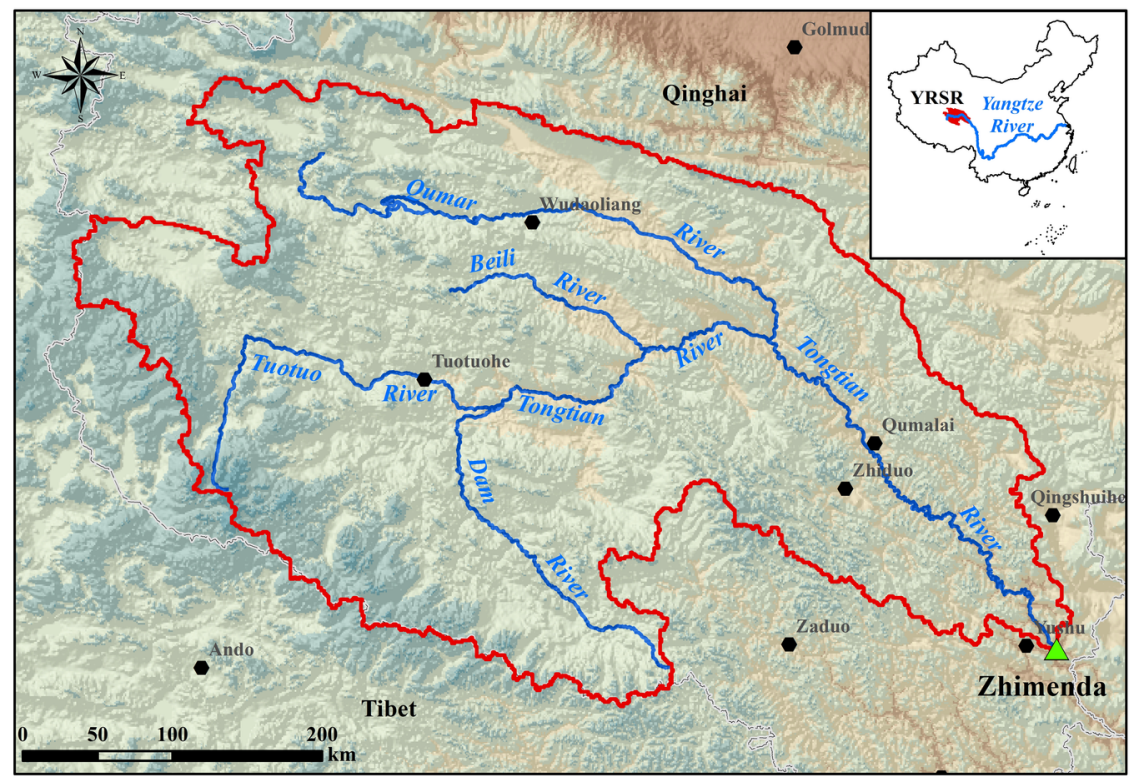

Legend

$\triangle$ Hydrologic station $\bullet$ Meteorological station - River $\square-\square$ Province $\square$ SRYR Elev.(m)

$\square<3500 \square 3500$ to $4000 \square 4000$ to $4500 \square 4500$ to $5000 \square 5000$ to $5500 \square>5500$

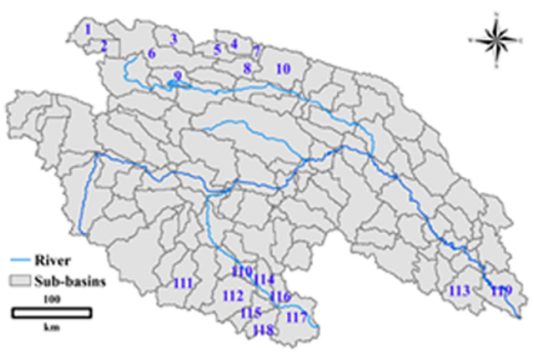

Num. of sub-basins: 119

Threshold drainage area: $80 \mathrm{~km}^{2}$

Num. of Hydrological response units:265
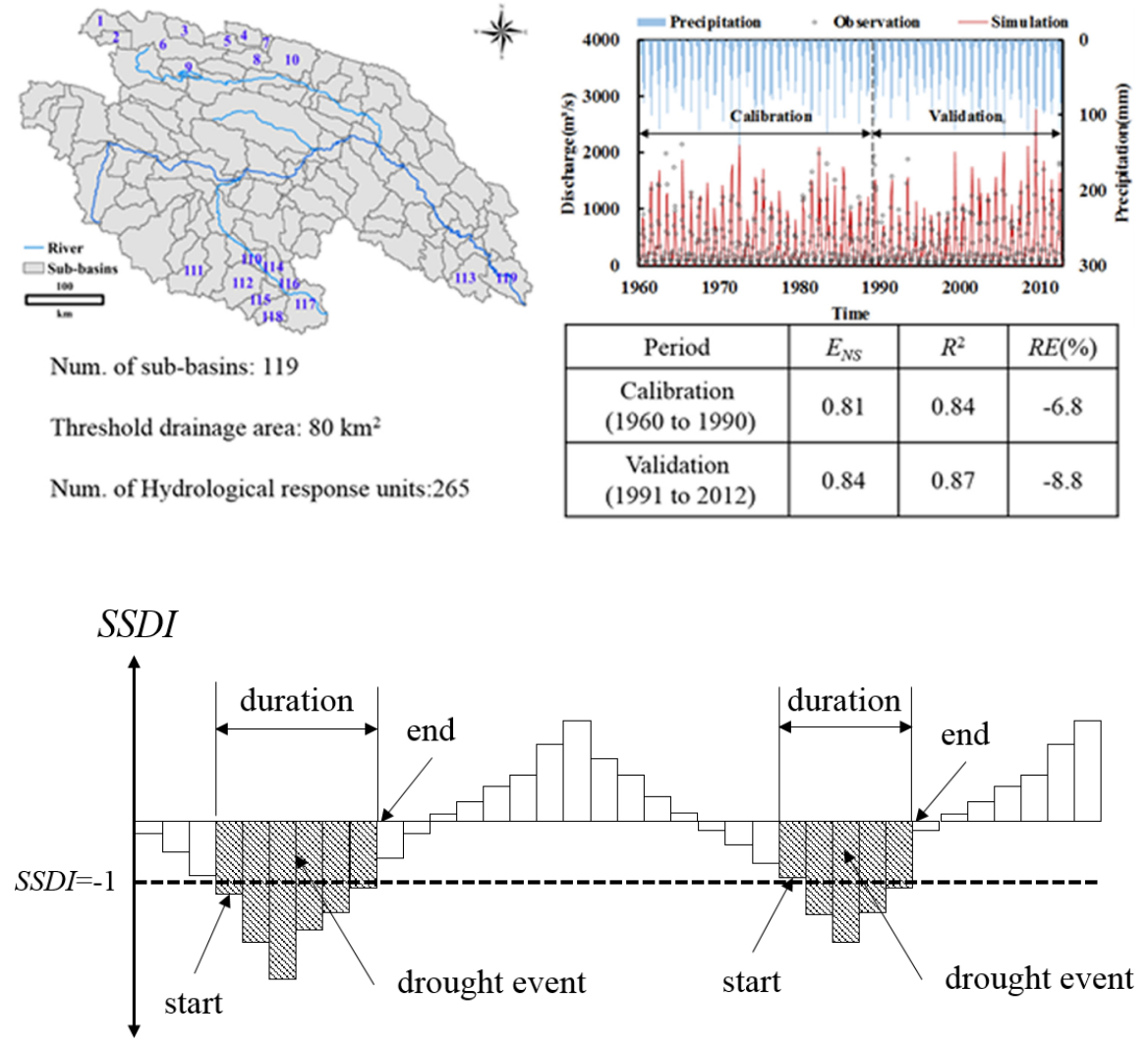


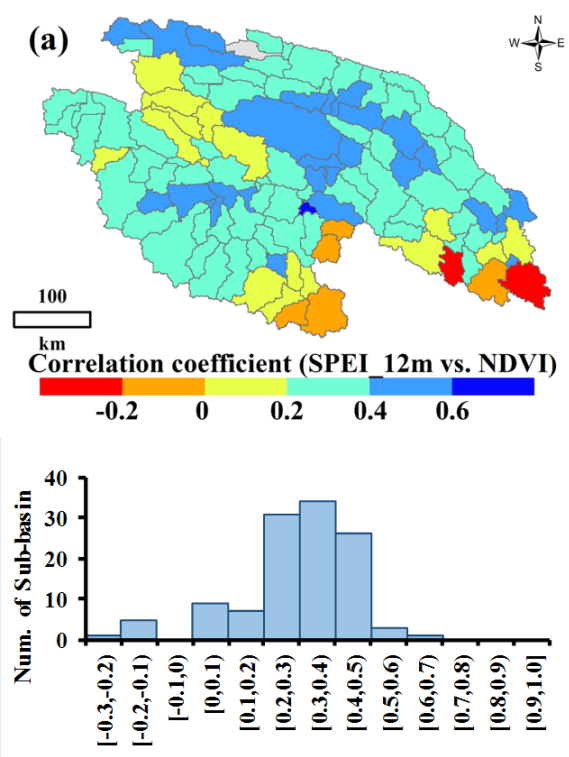

Correlation coefficient

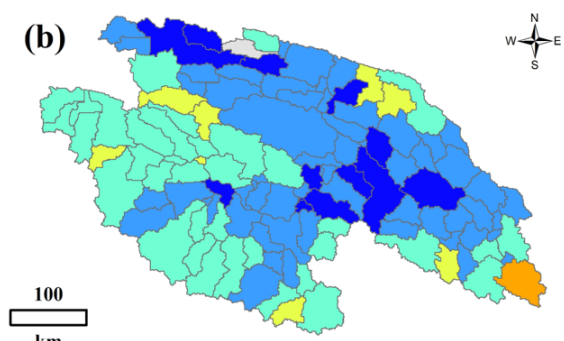

Correlation coefficient (SSDI_12m vs. NDVI)

$\begin{array}{lllll}-0.2 & 0 & 0.2 & 0.4 & 0.6\end{array}$

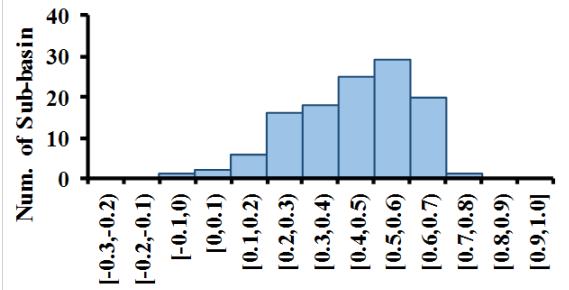

Correlation coefficient
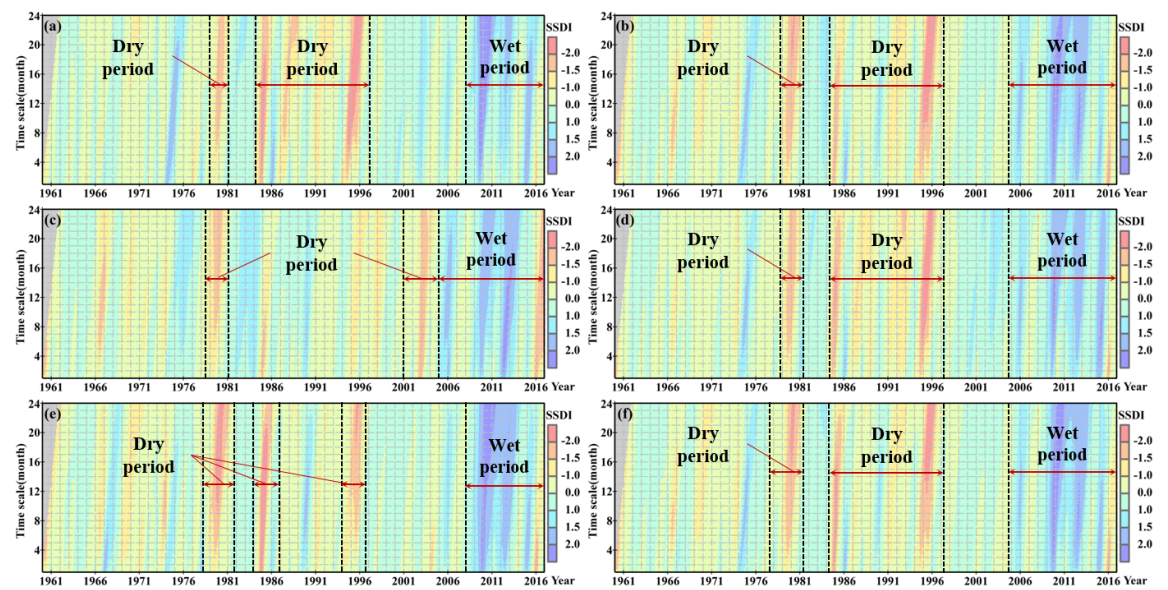
(a)

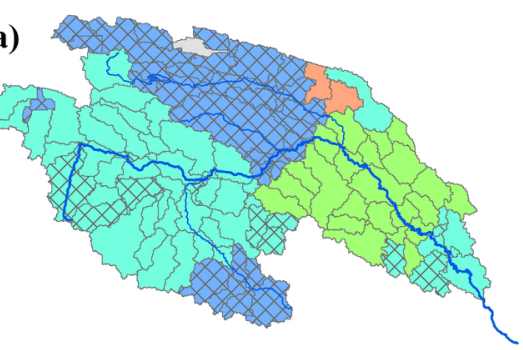

(b)

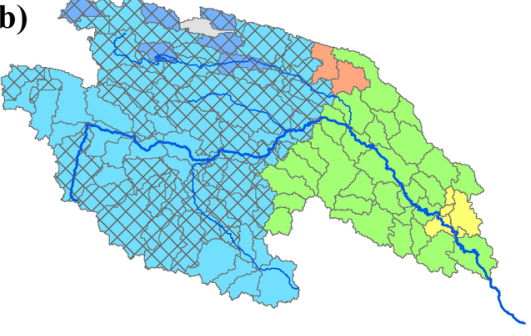

(d)

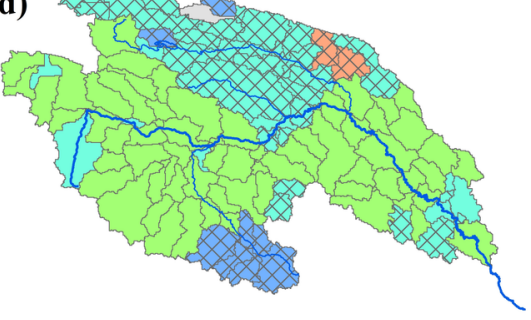

$w-p^{n}=$

Trend of SSDI values

$\square<-0.01$

$\square-0.01$ to 0

$\square 0$ to 0.01

$\square 0.01$ to 0.02

$\square 0.02$

$5 \otimes$ Significant at the $95 \%$ confidence level

$\begin{array}{llll}0 & 75 & 150 & 300 \\ & & & \end{array}$

(c)

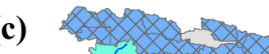

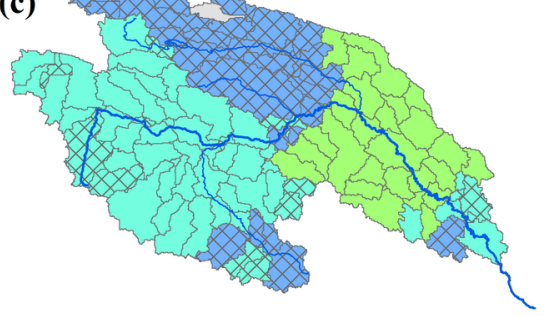

(e)

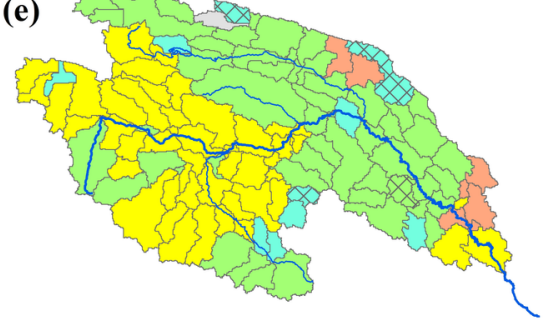



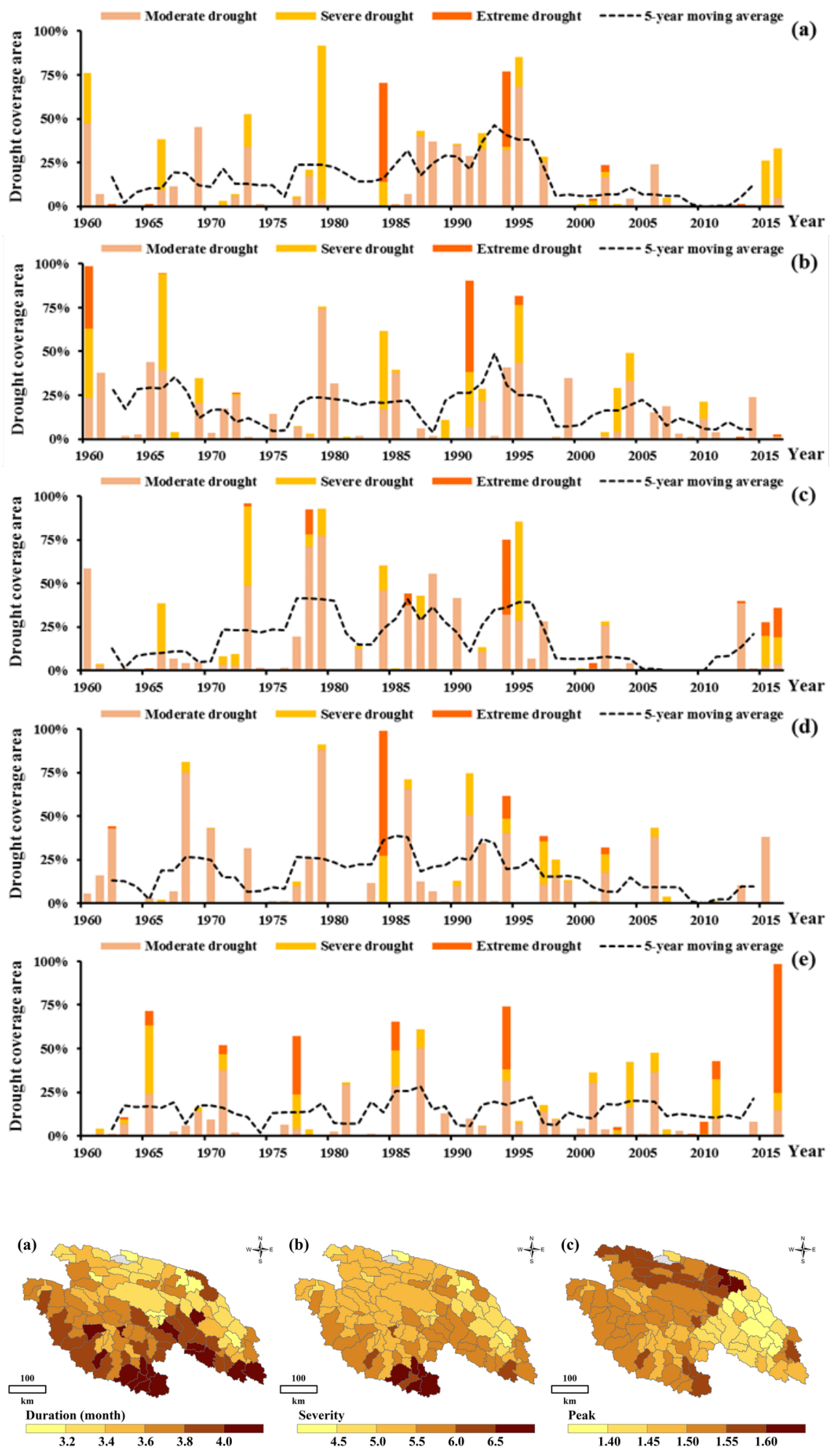

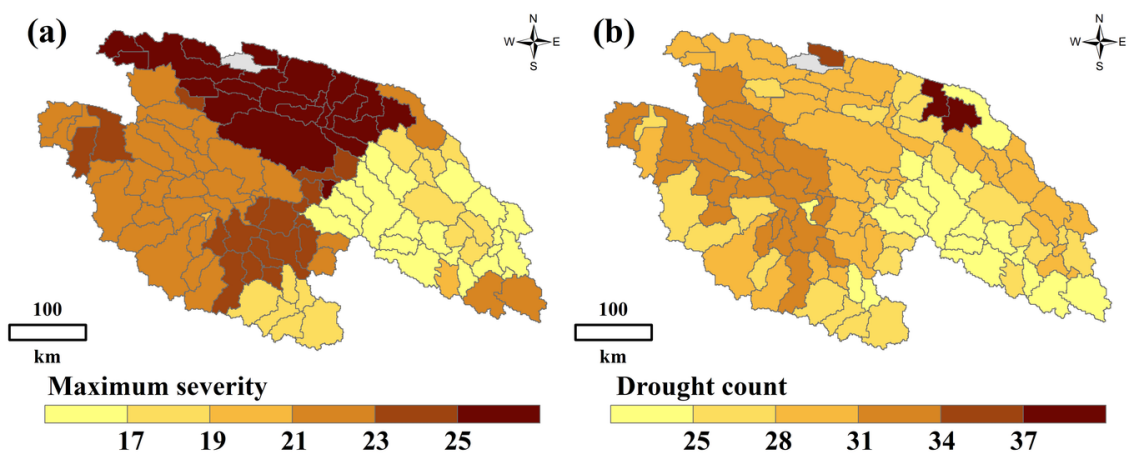

Drought count $\begin{array}{lllll}25 & 28 & 31 & 34 & 37\end{array}$ 

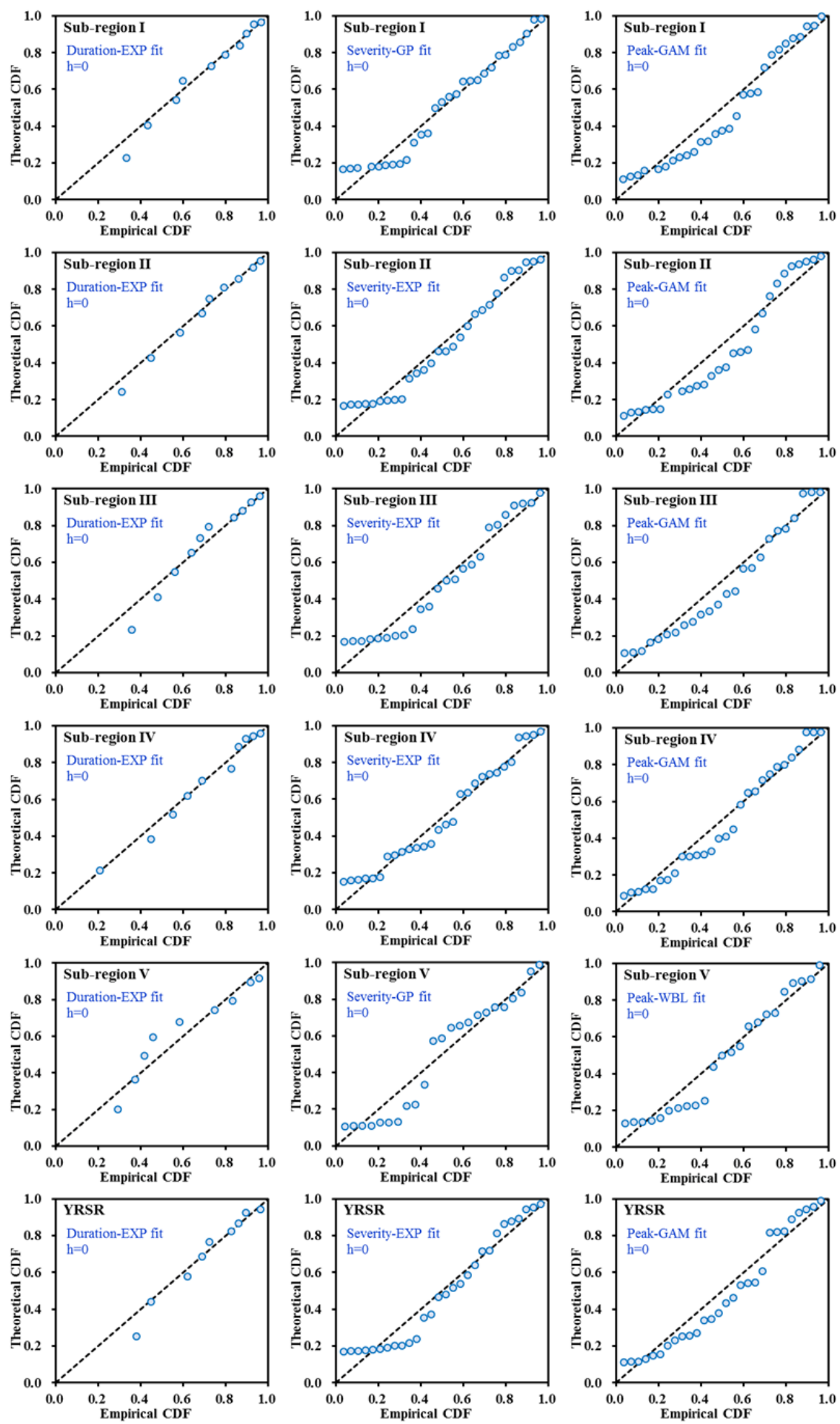

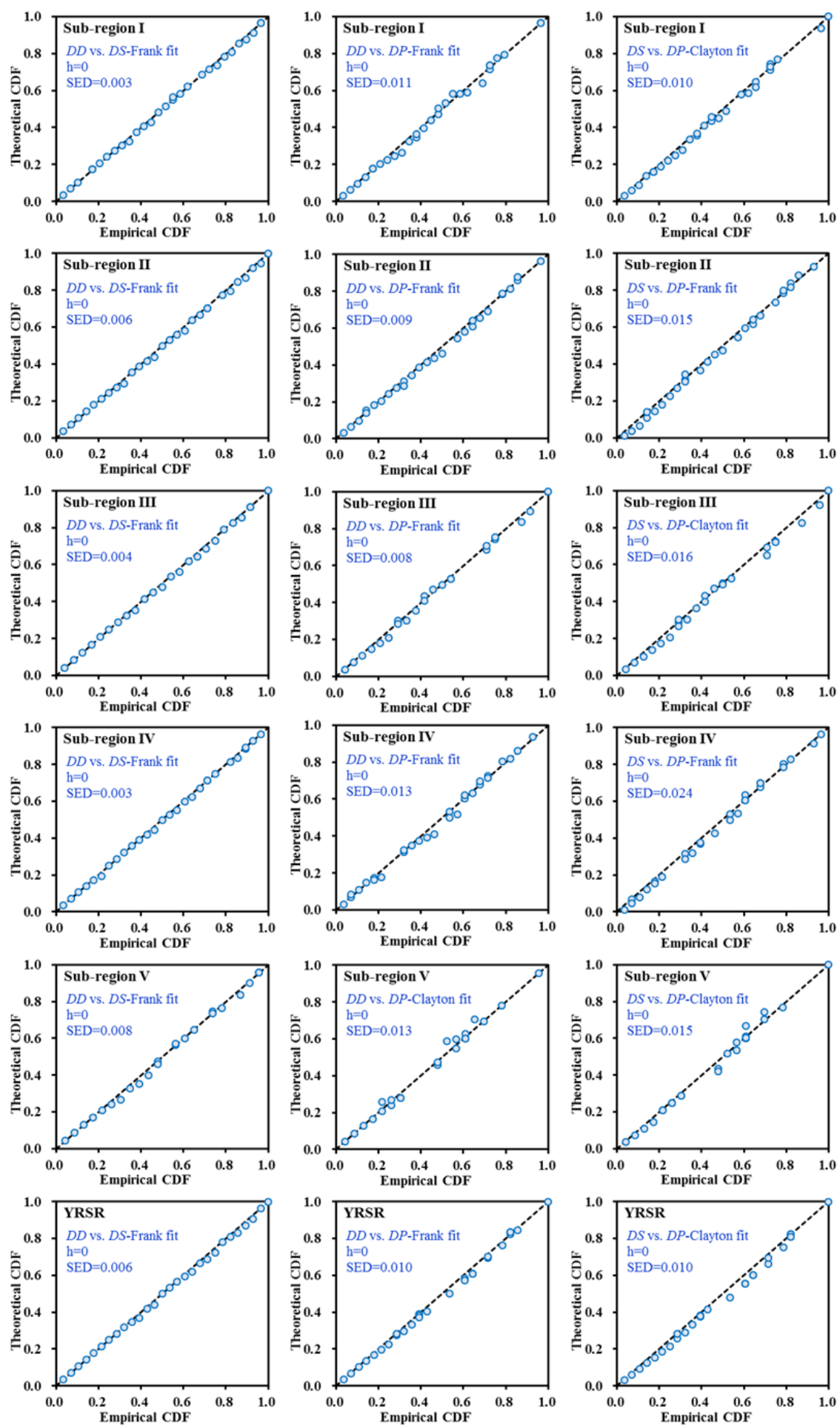

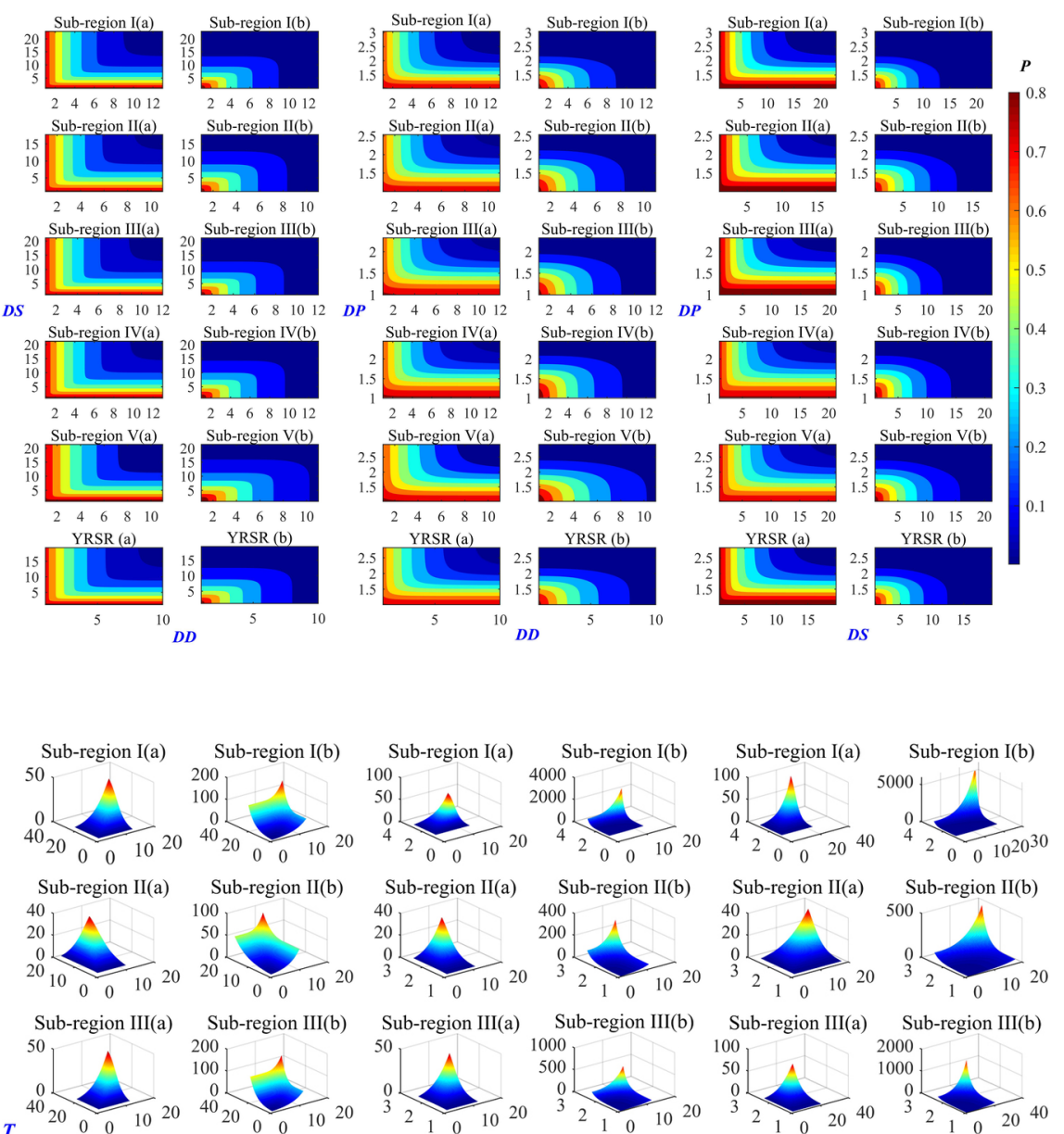

Sub-region III(a)
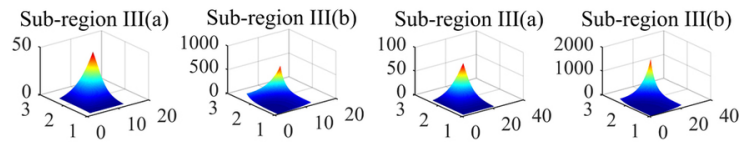

Sub-region IV(a)

Sub-region IV(b)

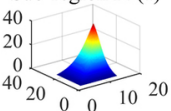

200
100
0

Sub-region IV(a)

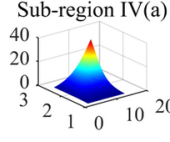

Sub-region IV(b)

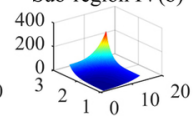

Sub-region IV(a) Sub-region IV(b)

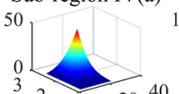

500 .

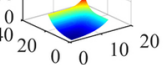

Sub-region V(b)

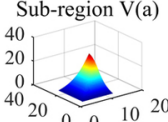

Sub-region $\mathrm{V}(\mathrm{b})$

Sub-region V(a)
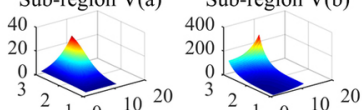

Sub-region V(a)

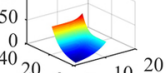

$\underbrace{0}_{2} \underbrace{}_{10}$

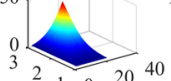

1000
500.

$$
\text { YRSR(a) }
$$

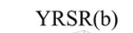

YRSR(a)
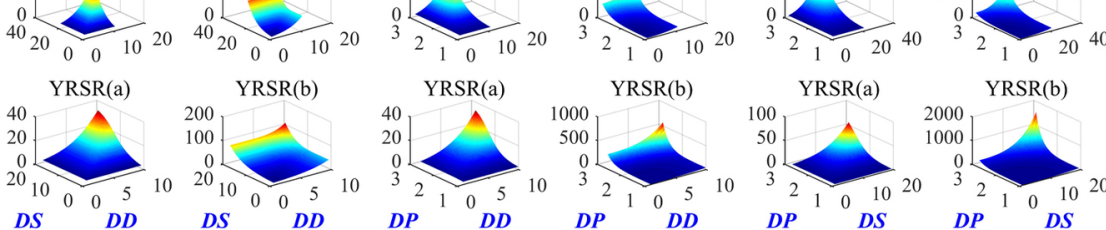

YRSR(b)

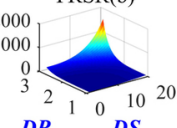




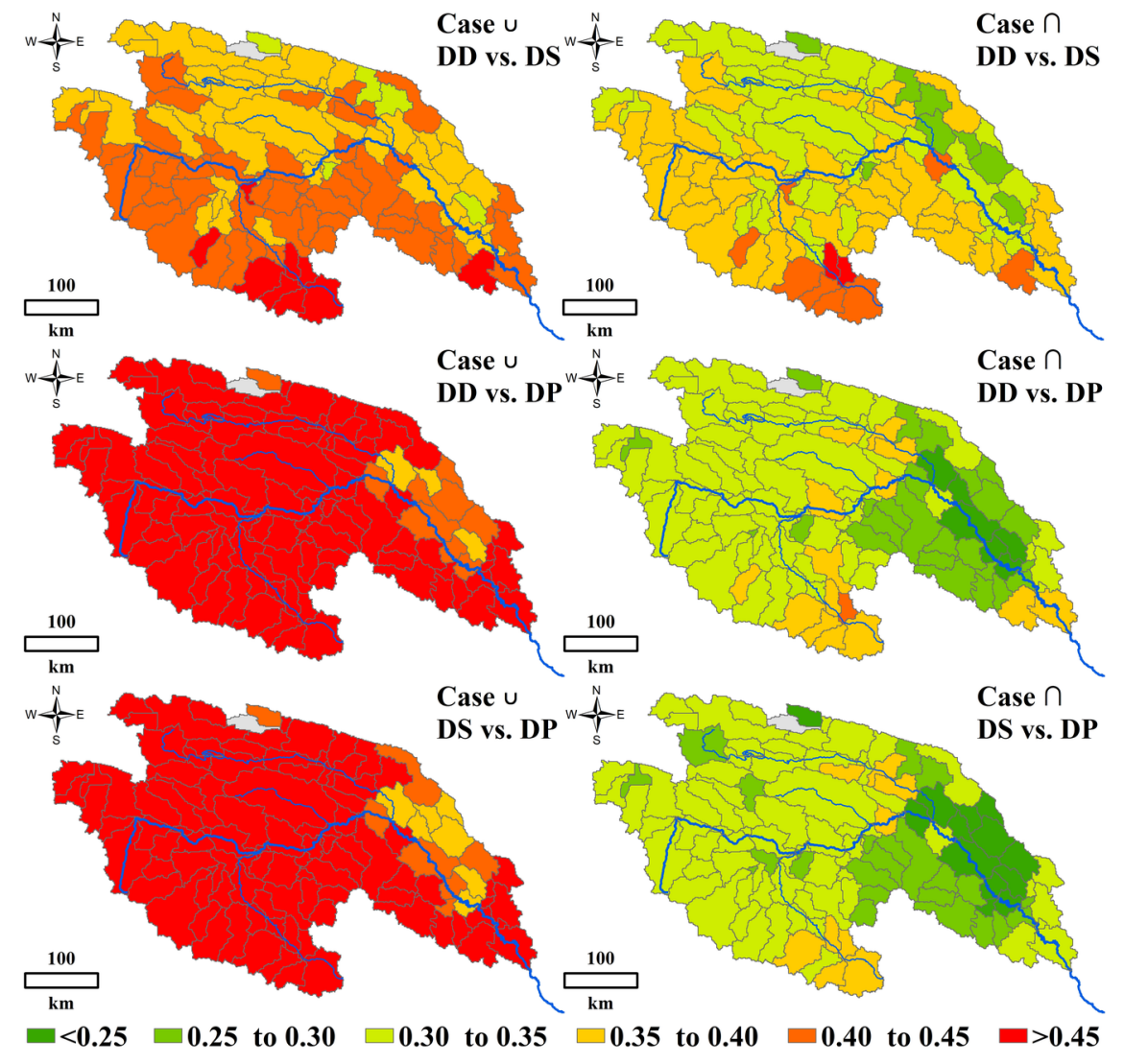



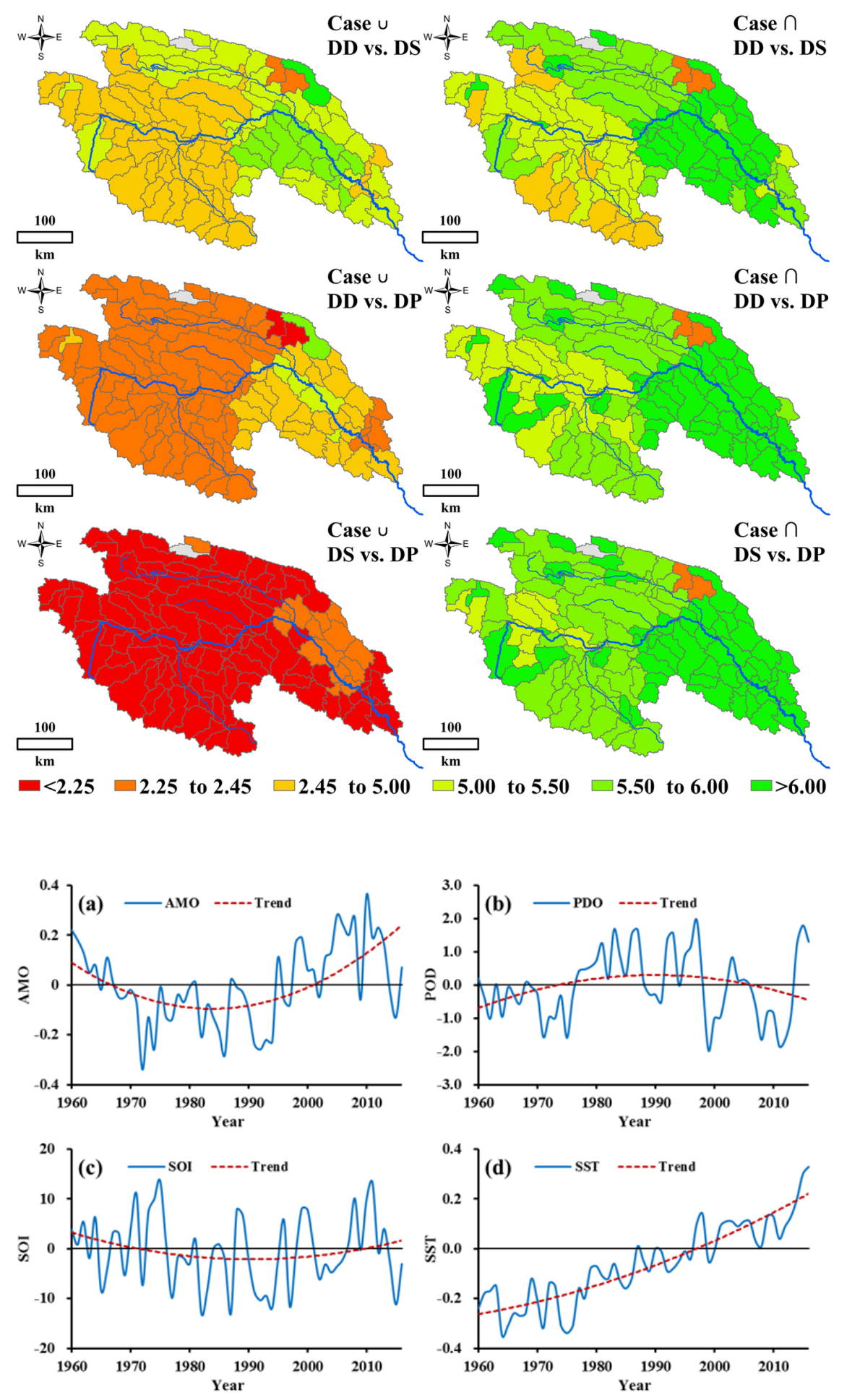

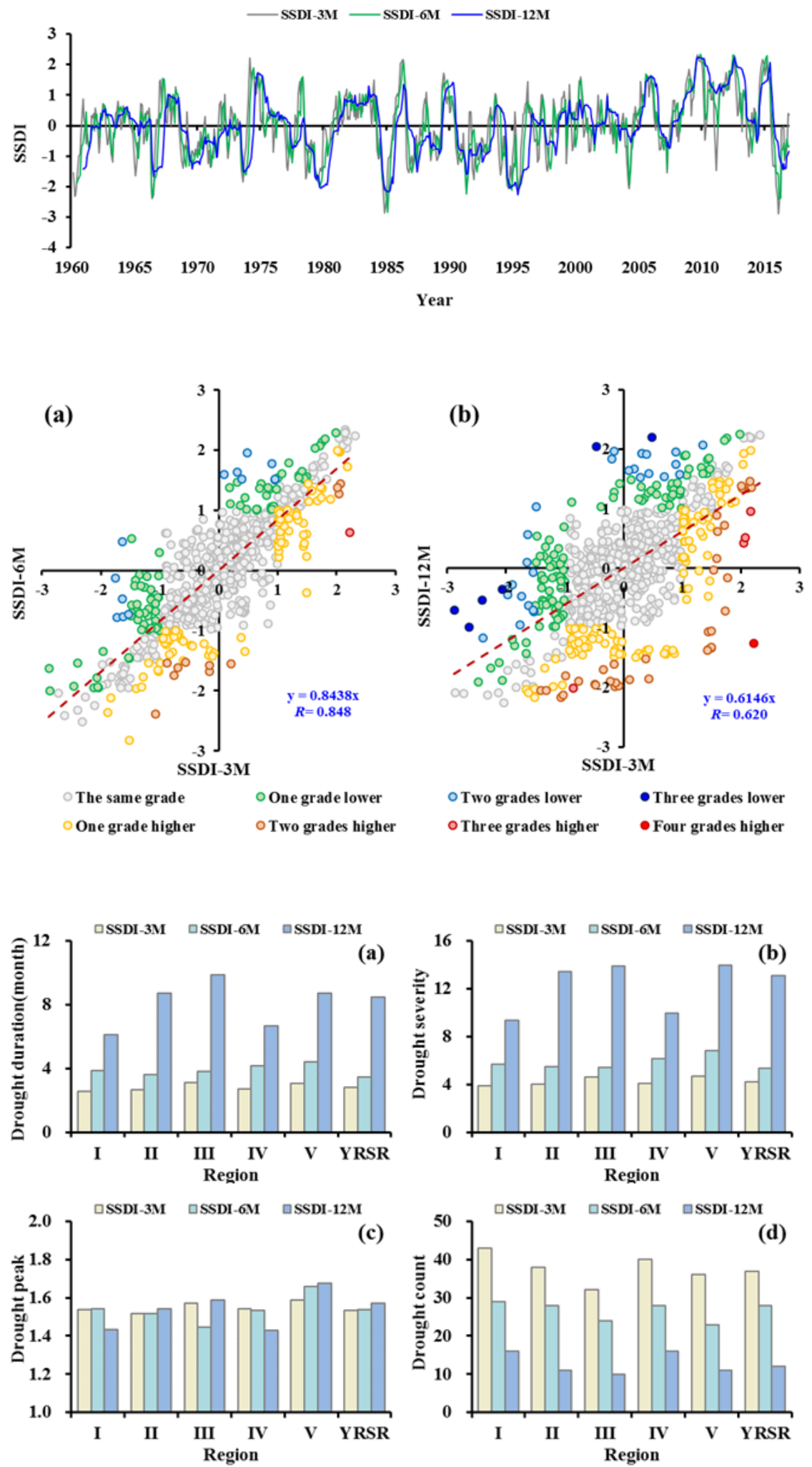

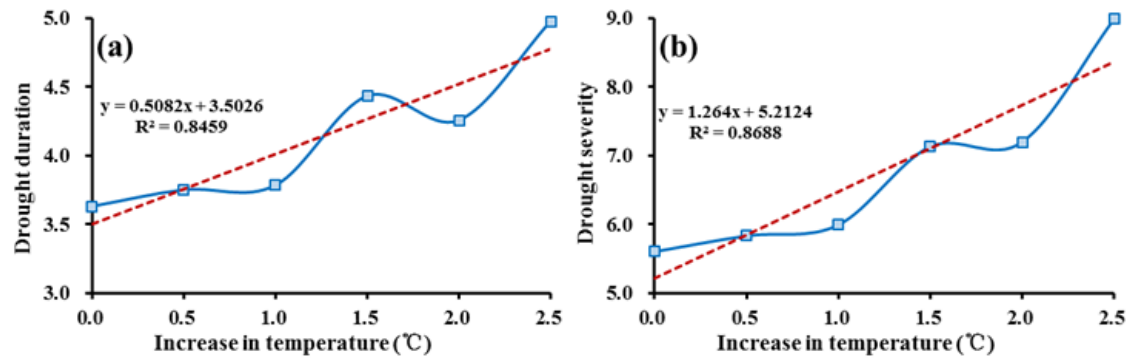